\title{
Intonational correlates of Subject and Object realisation in Mawng (Australian)
}

\author{
Janet Fletcher, Hywel Stoakes, Ruth Singer, Deborah Loakes \\ School of Languages and Linguistics, University of Melbourne, Australia \\ janetf; hstoakes; rsinger; dloakes@unimelb.edu.au
}

\begin{abstract}
A range of intonational devices can be used in the grammar of information and corrective focus marking in languages with relatively free word order. In this paper we explore whether nouns in the Australian Indigenous language Mawng are realised differently depending on syntactic function and focus. Results show that the pitch level associated with Subjects is higher in conditions of corrective focus compared to other utterance contexts and there is a strong correlation between focus and utterance position. Placing a word in a corrective focus context does not appear to have an effect on word duration in this corpus confirming that pitch register variation and intonational phrasing are the major prosodic cues associated with corrective focus in Mawng.
\end{abstract}

Index Terms: Intonation, information structure, Australian Indigenous Languages

\section{Introduction}

Descriptions of prosody and intonation in Australian Indigenous languages [e.g. 7] suggest that they are head/edge prominence languages rather than head prominence or edge prominence languages according to a current model of prosodic typology [8]. Intonational pitch accents aligned with rhythmically prominent syllables of words, combine with phrase tones to demarcate the left and right edges of higherlevel prosodic constituents i.e. Intonational Phrases (IPs). It is well known that phonetic and phonological devices that mark out these heads and/or edges can also be linked to the grammar of pragmatic focus marking in languages. These devices might include combinations of the following: manipulations of phrase-level pitch range, intonational and prosodic phrasing, and manipulations of intonational prominence, including the use of different types of pitch accents for contrastive emphasis [1]. Languages can de-accent material (i.e. reduce the number of pitch accents in a phrase) and/or de-phrase non-focal material (i.e. reduce the number of intonational constituents) to promote a particular kind of discourse interpretation. Interestingly, languages with diverse word-level prosodic characteristics like Georgian, Mandarin, or Chichewa also tend to show post-focal pitch range compression, with prosodic phrasing or lexical tonal contrasts (in the case of Mandarin and Chichewa) left intact. It has also been argued that languages with relatively free word order use deliberate intonational devices to signal different information structure categories such as topic or focus i.e. intonation patterns are not just a reflex of word order manipulations or position in utterance, for example.

Relatively few studies have examined the prosody and information structure interface in Australian Indigenous languages with some notable exceptions (e.g. [2], [3], [4], [5]). In Mawng, the language under investigation in this study, corrective focus on noun objects can be expressed grammatically in at least two ways. The object must be overt (obligatory), and left-dislocation of the noun may also be observed (which is less common) [6]. This phenomenon has also been noted in a range of other Australian languages (e.g. Warlpiri, Arrernte, Dalabon), as well as in many other so called "free word order" languages in the world.

There are also intonational patterns that are typical of object "fronting" in Mawng. In an earlier experiment that examined the intonational realization of corrective focus in Object Verb constructions, it was noted that a fronted object is usually realised in higher pitch register and is produced in a tonally marked accentual phrase, with clear post-focal pitch compression of the following verb [7]. However, it remains to be shown how Subjects are realised when they are in focus. In this paper, we present the results of an experiment designed to elicit corrective focus in SVO constructions to complement our earlier studies of $\mathrm{OV}$ word order to see whether intonational patterns observed in our earlier experiment extend across different word order conditions.

\subsection{Aims of study}

The following predictions were made on the basis of previous findings for Mawng and for other "free word order" languages. If there is a deliberate manipulation of intonational parameters to realize corrective focus, we predict that this could manifest either as a specific accent type (e.g. $\left.\mathrm{L}+\mathrm{H}^{*}\right)$ on the corrective focused subject with a strong lead tone or an upstepped simple ${ }^{\wedge} \mathrm{H}^{*}$ accent which is realised in the highest part of a speaker's pitch range. We also predict intonational phrasing patterns that are similar to $\mathrm{OV}$ constructions in Mawng under conditions of corrective focus. The initial focussed subject may be realised as a separate intonational phrase, separated from the verb by a tonally marked prosodic boundary. Given these prosodic strategies, it remains to be seen whether there is deaccenting or dephrasing of post-focal material typical of many stress accent languages when information focus is on initial Subjects. However our results to date suggest we may see a pattern similar to that of Italian, for example, where the accent pattern remains intact in post-focal utterance contexts. Finally, we wanted to investigate whether there is additional lengthening of Subjects under conditions of corrective focus given that there may be durational adjustments associated with accentual prominence as found in a previous study of Mawng [9]. Moreover if focussed Subjects are realised as separate intonational phrases we might also expect additional duration differences due to pre-boundary lengthening of the focal subject. 


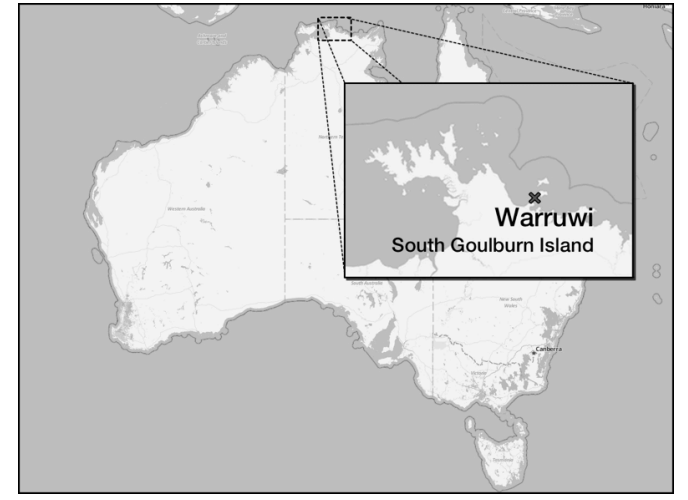

Figure 1: Map of Australia showing where the Indigenous language Mawng is spoken (source: open street map, 2015).

\section{Materials and Method}

\subsection{Mawng}

Mawng is a language of the Iwaidjan family spoken in the community of Warruwi on South Goulburn Island off the coast of Northern Australia (see Figure 1. for the approximate location). There are around three hundred speakers of the language and it is one of the handful of Indigenous languages that is still being acquired by children as a first language. Mawng is a mildly polysynthetic language which allows multiple word orders including SVO, OVS, OV, VSO which is typical of most Australian languages. It is traditionally described as a stress language and our earlier studies [7] suggest that the prosodic typology of Mawng is like other languages of the region and is best characterized as a head/edge prominence language with major prominencelending pitch movements docked to a word accent that denote the left edge of an accentual phrase and right-edge tones that often delimit the edge of a phonological word.

\subsection{Speakers and language materials}

The corpus was recorded during two field trips at Warruwi, Goulburn Island, Northern Territory. Four adult female speakers of Mawng (NN, RM, NG, and SM) participated in the speaking tasks. A mini-dialogue was constructed to elicit neutral, information and corrective focus on Subjects. The verb was also modified to have a transitive versus intransitive reading although we only present results for the transitive corpus in this paper. An example of the dialogue using a transitive verb construction is shown below with the Subject token(s) of interest highlighted in bold font showing 15 different fish names. Part A is the "neutral" focus condition and part $\mathrm{C}$ the corrective focus utterance. Part $\mathrm{B}$ is the negation utterance where we might predict that the Subject is no longer in focus, with the $\mathrm{VO}$ in information focus. Unlike our previous experiments ([7]) word order of PART C was the produced with the more marked SVO rather than OV word order. Our interest was primarily on the intonational realisation of Subject focus in the corrective focus utterance C (utterance initial) with respect to the preceding negation utterance (context B) where the Subject is realised in utterance-final position, and possibly in a post-focal context.

Three speaker pairs produced the mini dialogue up to three times. One speaker produced part A and a second speaker produced parts B and C. All parts of the dialogue were labeled and transcribed orthographically and a grammatical analysis and translation was annotated in ELAN. Files were converted from ELAN to Praat, and then further converted into EMU $[10,11]$. An intonational analysis was performed and F0 contours were annotated using a tone-target analysis. Major pitch movements corresponding to pitch accents and accentual and intonational phrase boundaries were labelled using the F0 signal and auditory analysis. Three kinds of pitch accent labels were used in this study, ${ }^{\wedge} \mathrm{H}^{*}$ or $\mathrm{L}+{ }^{\wedge} \mathrm{H}^{*}$ representing a clear upstep of pitch level of the accent relative to surrounding material, $\mathrm{H}^{*}$ which is the "default high" pitch accent sometimes preceded by a shallow rise, and $! \mathrm{H}^{*}$ which represents a lowered pitch peak relative to a preceding $\mathrm{H}^{*}$ accent. An example of an annotated pitch contour for the corrective focus condition (C) is shown in Figure 2. The initial word wirlmu "barramundi" is the Subject of the utterance and receives a relatively high ${ }^{\wedge} \mathrm{H}^{*}$ pitch accent relative to the following verb.

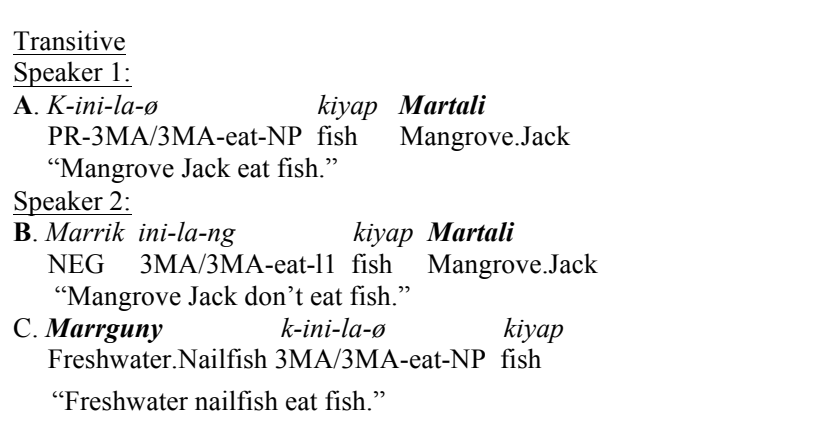

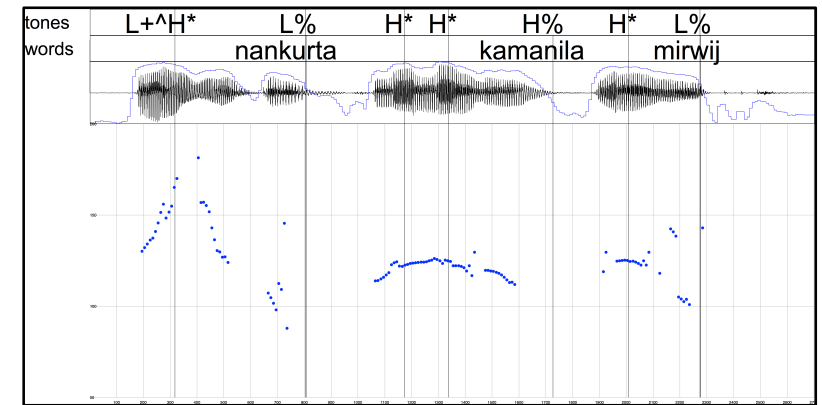

Figure 2. Speech waveform, F0 contour of an utterance produced by speaker RM of a Corrective focus (C) utterance showing a high pitch accent $\mathrm{L}+{ }^{\wedge} \mathrm{H}^{*}$ and right-edge $\mathrm{L} \%$ boundary tone on the initial Subject nankurta "mullet.type".

All instances were noted in the Corrective focus context $\mathrm{C}$ where, a) the Subject was realised with an extreme pitch excursion on the accented word; b) the Subject was realised in a separate accentual phrase or intonational phrase; c) the Object was also realised with a pitch accent. Part B of each mini dialogue was also annotated to see how the Subject and Object were realised in the negation utterance. We chose to focus initially on Parts B and C because the same speaker produced the tokens of interest. A total of 772 Subject and Object tokens were analysed across the two parts of the dialogue, B and C. Some tokens were discarded due to mistracking of the F0 trace or where speakers made mistakes or misread the carrier phrase. Verbs were also intonationally annotated. $\mathrm{F} 0 \mathrm{~Hz}$ values were extracted for tone targets using EmuR, and then converted to semitones using a formula with a base frequency of $50 \mathrm{~Hz}$ after [11]. Duration values were also extracted for the entire subject and object tokens in contexts B 
and C. A linear mixed effects analysis was then performed using R and lmer [12] to test the relationship between pitch level in semitones of the pitch accent, the duration of the noun, and utterance position (part B post-focal, and part C, corrective focus), and syntactic structure (whether the noun was a subject or object). A maximally specified mixed model was implemented with the fixed factors focus and syntactic structure (Subject, Object) and with random factors, speakers and word specified for both intercept and slope.

\section{Results}

\subsection{Tonal marking of Subjects and Objects}

Figures 3. and 4. plot the distribution of the major pitch accent types that were annotated for Subjects and Objects across the corpus in the negation (B) and corrective focus (A) utterances. As predicted all speakers produced pitch accents on sentential Subjects in the corrective focus utterance. In fact all Subjects and Objects were pitch accented in this corpus. Recall that in context B the word order is VOS and in Part C, the word order is SVO so subjects are utterance-final in the B context but utterance- initial in the $\mathrm{C}$ context.

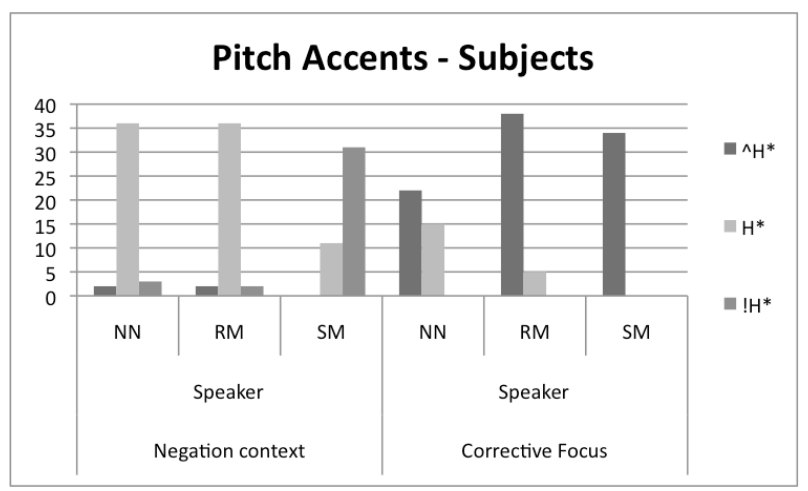

Figure 3. Pitch accent distribution for SUBJECTS in parts B (negation) and $\mathrm{C}$ of the corrective focus condition for three speakers.

Most speakers produced an upstepped pitch accent $\left({ }^{\wedge} \mathrm{H}^{*}\right)$ on sentence initial subjects in the Corrective Focus utterance as predicted. Interestingly, these were not always realised with a strong lead tone as shown in the example in Figure 2. By contrast when the Subject was utterance final and in post-focal position in context B, speakers tended to produce a "normal" $\mathrm{H}^{*}$ accent or a downstepped ! $\mathrm{H}^{*}$ relative to the preceding verb so there was no evidence of de-accentuation. In the negation utterance, the highest pitch accent in the utterance was mostly reserved for the utterance-initial verb which we assume was in focus in the negation utterance.

While not shown here, Subjects in context C (Corrective Focus) were also often realised as a separate accentual phrase or in some cases as separate major intonational phrases, often separated by a pause from the following verb. For example $93 \%$ of Subjects produced by speaker RM were realised as separate intonational phrases. Speaker SM produced $56 \%$ and Speaker NN 59\% as separate intonational phrases with the remainder realised as accentual phrases with a minor phrase boundary. All speakers showed more or less a similar pitch accent pattern in the Corrective focus utterances although speaker NN did not always produce a higher pitch accent relative to the following verb. In the negation utterance (B), speakers $\mathrm{SM}$ and $\mathrm{RM}$ produced a combination of $\mathrm{H}^{*}$ or $! \mathrm{H}^{*}$ accents on Subjects (which were utterance final in this context).

In the negation utterance, there was no evidence of deaccentuation with all objects receiving mostly a $\mathrm{H}^{*}$ or $! \mathrm{H}^{*}$ pitch accent relative to the preceding verb. In a few cases, accents were actually "upstepped" relative to the verb particularly if the following Object was realised as a new intonational phrase. Speaker SM tended to produce the Verb and Object as a single intonational phrase and routinely produced a downstepped pitch accent on the Object in this context. Speakers RM and NN by contrast often paused after the verb so not so many downstepped accents were produced on Objects. Objects in the corrective focus utterance (C) were also produced with greater variation in pitch level by speaker NN whereas speaker RM like SM tended to produce mostly ! $\mathrm{H}^{*}$ on final objects.

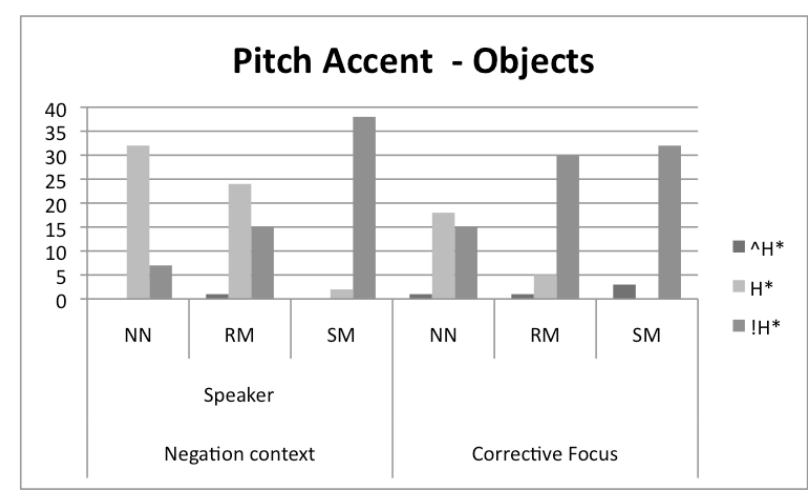

Figure 4. Pitch accent distribution for OBJECTS in parts B (negation) and $\mathrm{C}$ of the corrective focus condition for three speakers.

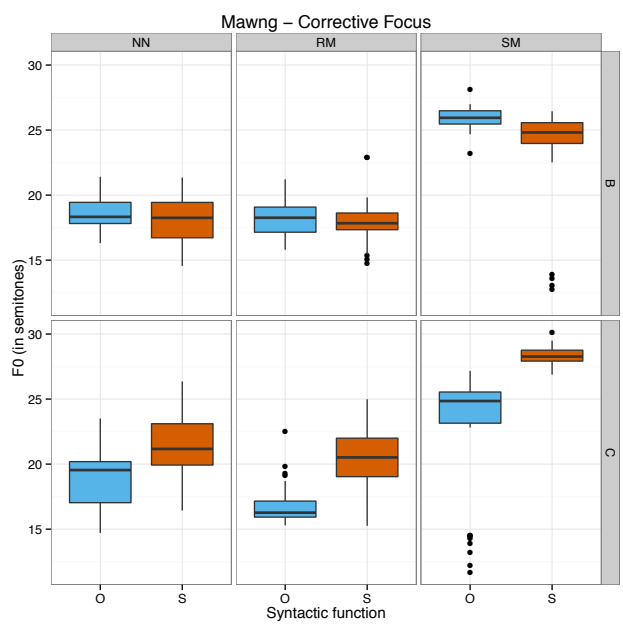

Figure 5. Pitch level in semitones of pitch accents in Subject (S) and Object $(\mathrm{O})$ contexts in parts $\mathrm{B}$ and $\mathrm{C}$ of the corrective focus condition for all speakers. Within each panel, Object tokens are on the left, Subject tokens on the right. 


\subsection{Pitch scaling of subjects and objects}

Figure 5. plots pitch level in semitones associated with all pitch accent tone targets produced by the three speakers in the two focal contexts. Results of the lmer and subsequent likelihood ratio test showed that there is a significant interaction between SYNTACTIC CATEGORY (subject versus object) and FOCUS position (corrective focus versus post-focal) $\left(\chi^{2}=24.999 .437, \mathrm{p}<0.001\right)$. As can be seen from Figure 5 (bottom panel), pitch accents are realised at a higher pitch level (measured in semitones) in corrective focused Subjects compared to non-focal Objects in the same utterance for all three speakers, concurring with the intonational analysis presented above. Subjects are also realised at a higher pitch level compared to Subjects in the negation utterance where they are utterance final and not utterance initial and are postfocal.

A series of post-hoc Tukey tests confirmed there is a strong interaction between syntactic function and focus position and that there is a significant pitch height difference of around $4.5 \mathrm{st}$ between Subjects that are in the corrective focus versus negation utterance across the corpus $(\mathrm{p}<0.001)$. There are no significant pitch level differences for Objects in either context, or Subjects versus Objects in the negation utterance - B ( $>0.01)$. The most likely explanation for this is that Subjects and Objects are both post-focal in the negation utterance with the "highest" pitch accent of the utterance realised on the utterance initial verb in these VOS constructions.

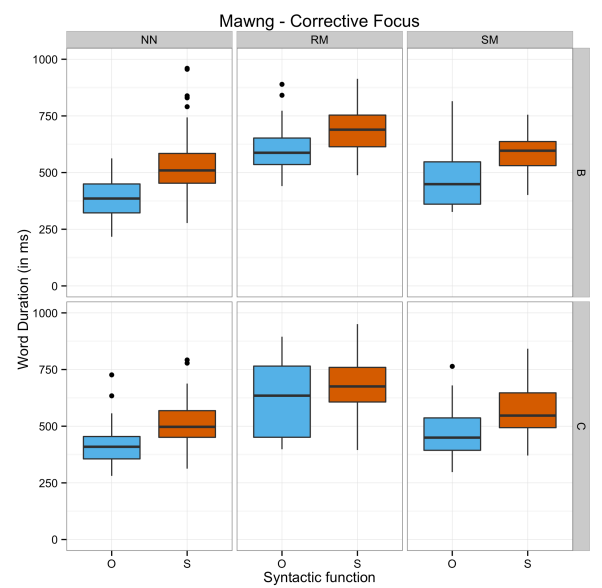

Figure 6. Word duration (ms) of Subjects $(\mathrm{S})$ and Objects $(\mathrm{O})$ in parts $\mathrm{B}$ and $\mathrm{C}$ of the corrective focus condition plotted for all speakers.

\subsection{Word duration}

Figure 6. plots overall word duration in sentence initial (Subject) and sentence final (Object) contexts in parts B and C of the focus construction. As can be seen quite clearly from the plots, there is no significant difference due to focus category $(p>0.01)$. In other words Subjects and Objects have similar durations whether they occur in the corrective focus condition or in the denial. Subjects in general are longer than Objects regardless $(p<0.02)$. This result could reflect word length differences between the Subject tokens which varied from two to five syllables in duration, and Objects which were mostly disyllabic in this experiment. Of particular note also is that Subjects are intonational phrase-final in the B context, whereas Objects are intonational phrase-final in the $\mathrm{C}$ context.
In other words, pre-boundary lengthening is most likely influencing these results and will be examined in future work.

\section{Discussion}

Our prediction that initial Subjects in corrective focus utterances are produced by Mawng speakers with an extrahigh pitch accent (i.e. using an expanded tonal space) relative to post-focal material, was largely borne out by the analysis presented in this paper. It was also the case that differences in pitch level between focussed and non-focal Subjects were highly significant across the corpus. These results concur with earlier findings that showed that utterance initial Objects in corrective focus are also realised in an expanded tonal space relative to post-focal material or non-focal material [9]. Speakers did not always produce a strong lead tone with these extra high pitch accents (i.e. $\mathrm{L}+{ }^{\wedge} \mathrm{H}^{*}$ ). Corrective focused Subjects were also often produced as a separate intonational constituent, concurring with our preceding findings for Mawng and confirming the importance of edges as well as heads in the prosodic typology of the language. They also accord well with studies of intonation and information structure in other Australian languages e.g. Jaminjung [2] and are similar to focus-marking strategies found in many other languages [1].

There were no instances of post-focal de-accenting of Subjects or Objects in the negation utterance with final Subjects always receiving a pitch accent, although they were usually realised in a somewhat compressed pitch range relative to Subjects in the corrective focus utterance. This presumably indicates a strategy to differentiate the two contexts given that the same speaker produced the negation and corrective focus utterances. It is also important to recall that in the negation utterance, focus is likely to be on the Verb plus Object in these VOS constructions which constitutes new information relative to part A of the mini-dialogue. It is certainly the case (impressionistically) that two speakers often produced the Verb and Object as single intonational phrases but further analyses of the corpus (including an analysis of the neutral focus utterance A) are needed to confirm this.

It also could be argued that the patterns observed in this study are potentially a reflex of utterance-initial position in the sentence, which is well attested as a site of discourse prominence in many Australian languages e.g. [13]. Initial position in a discourse segment is generally associated with higher pitch register or upwards pitch range reset in many languages. It could be argued that the patterns observed in this and our earlier experiment were mostly due to the word order manipulations in our subject design. However, the intonation patterns we have observed in the experiments so far are somewhat different to those in the neutral utterance (context A), which suggests there may be deliberate pitch range expansion and phrasing strategies to place Subjects in corrective Focus in this experiment. These strategies may serve an enhancing and demarcative purpose in spoken Mawng discourse.

\section{Acknowledgements}

This research was supported by an Australian Research Council Discovery Project DP110100938 and the ARC Centre of Excellence for the Dynamics of Language. The fieldwork component was also partly funded by a University of Melbourne Faculty of Arts Research grant to Ruth Singer. 


\section{References}

[1] C. Féry, "Focus as prosodic alignment". Nat. Lang. Linguistic Theory 31:683-734, 2013

[2] C. Simard, The Prosodic Contours of Jaminjung, a Language of Northern Australia. Manchester: University of Manchester PhD, 2010

[3] S. Hellmuth, F. Kügler \& R. Singer "Intonational patterns, tonal alignment and focus in Mawng". Proceedings of the 16th ICPhS satellite workshop: Intonation phonology: understudied or fieldwork languages, Saarbrücken, Germany, 2007.

[4] R. Singer, "Information structure in Mawng: intonation and focus." Selected papers of the 2005 conference of the Australian Linguistics Society, Allan, K. (ed). http://www.als.asn.au/, 2006.

[5] J. Bishop, Aspects of intonation and prosody in Bininj Gun-wok: An autosegmental-metrical analysis. $\mathrm{PhD}$ thesis, University of Melbourne, 2002

[6] R. Singer, Agreement in Mawng: productive and lexicalised uses of agreement in an Australian language Melbourne: University of Melbourne PhD thesis, 2006.

[7] J. Fletcher, R. Singer, D. Loakes, "Intonation and focus-marking strategies in Mawng." Tone and Intonation in Europe 5, Oxford, September 2012.

[8] S-A. Jun. "Prosodic typology by prominence type, word prosody \& macro-rhythm." Prosodic Typology II, Oxford:OUP, 520-39, 2014

[9] J. Fletcher, H. Stoakes, D. Loakes, R. Singer. "Accentual prominence and consonant lengthening and strengthening in Mawng". In The Scottish Consortium for ICPhS 2015 (Ed.) Proceedings of the 18th International Congress of Phonetic Sciences. Glasgow, UK: the University of Glasgow. ISBN 9780-85261-941-4. Paper 837 retrieved from http://www.icphs2015.info/pdfs/Papers/ICPHS0837.pdf, 2015

[10] R. Winkelmann, et al., emuR vsn 0.0.10. Available from: https://github.com/IPS-LMU/emuR, 2015.

[11] H. Quené. "Package "hqmisc"" Available from https://cran.rproject.org/web/packages/hqmisc/index.html, 2014.

[12] D.Bates, M.Maechler, B. Bolker, S. Walker "Fitting Linear Mixed-Effects Models Using lme4", Journal of Statistical Software, 67(1), 1-48. doi:10.18637/jss.v067.i01, 2015.

[13] J. Simpson and I. Mushin, "Clause-initial position in four Australian languages. In Mushin, Ilana \& Brett Baker (eds.) Discourse and grammar in Australian languages". Amsterdam: John Benjamins. 25-57, 2008. 